\title{
Simulation of satellite lidar and radiometer retrievals of a general circulation model three-dimensional cloud data set
}

\author{
M. Doutriaux-Boucher ${ }^{1}$ J. Pelon, ${ }^{2}$ V. Trouillet, ${ }^{2}$ G. Sèze, ${ }^{3}$ H. Le Treut ${ }^{3}$ \\ P. Flamant, ${ }^{4}$ and M. Desbois ${ }^{4}$
}

\begin{abstract}
The inclusion of a backscatter lidar on a space platform for a radiation mission, as proposed by various space agencies, aims to bring new information on three-dimensional cloud distribution, with a special emphasis on optically thin cirrus clouds, which are presently poorly detected by passive sensors. Key issues for such cloud observational studies are the detection of multilayered cloud systems, thin cirrus, and fractional cloud cover, knowledge that would improve our understanding of the global radiation budget. To assess the impact of such lidar measurements on cloud climatology, a 1 month cloud data set has been simulated with a general circulation model (GCM). The cloud detection capability of a spaceborne scanning backscatter lidar is assessed with the use of two detection schemes, one based on limitations in the detected cloud optical depth and the other based on lidar signal-to-noise ratio. The cloud information retrieved from passive radiometric measurements using a procedure like that used in the International Satellite Cloud Climatology Project is also simulated from the same GCM cloud data set. It is shown that a spaceborne backscatter lidar can improve significantly the retrieval of thin cirrus clouds as well as underlying cloud layers. High-level cloud retrieval from a spaceborne lidar therefore appears as a powerful complement to radiometric measurements for improving our knowledge of actual cloud climatology.
\end{abstract}

\section{Introduction}

Cloud cover and cloud properties are key parameters of the climate system and need to be monitored adequately for us to understand present-day climate and future climate change. The International Satellite Cloud Climatology Project (ISCCP) was designed to build a global cloud database from passive satellite radiometry [e.g., Schiffer and Rossow, 1983; Rossow and Schiffer, 1991]. This data set provides a global distribution of cloud parameters, such as cloud optical depth and cloud top pressure, from 1983 up to now and has led to considerable progress regarding the description of spatial and temporal cloud distributions [Rossow et al., 1989; Rossow and Lacis, 1990], deep convective systems [Del Genio and Yao, 1987], relevant cloud microphysics [Han et al., 1994], or cloud diurnal cycle [Cairns, 1995].

\footnotetext{
${ }^{1}$ Laboratoire d'Optique Atmosphérique, Université de Lille-I, Villeneuve d'Ascq, France.

${ }^{2}$ Service d'Aéronomie du CNRS, Université Paris 6, Paris, France.

${ }^{3}$ Laboratoire de Météorologie Dynamique du CNRS, Université Paris 6, Paris, France.

${ }^{4}$ Laboratoire de Météorologie Dynamique du CNRS, Ecole Polytechnique, Palaiseau, France.
}

Copyright 1998 by the American Geophysical Union.

Paper number 98JD02378.

0148-0227/98/98JD-02378\$09.00
However, some limitations and discrepancies with other remote-sensing techniques have been evidenced by comparative studies [Rossow et al., 1993; Liao et al., 1995a, b]. These limitations are generally associated with the complex three-dimensional (3-D) structure of real cloud fields. Other observational systems used in conjunction with the afore-mentioned passive radiometry techniques, are therefore required. A combination of high-resolution infrared radiometer sounder (HIRS) and advanced very high resolution radiometer (AVHRR) data is used to retrieve multilayered cloud systems [Baum et al., 1994, 1995]. High-cloud properties are retrieved from the Stratospheric and Gas Experiment (SAGE II) by an occultation technique [Liao et al., 1995a; br, Wang et ol :, 1996]. The cloud parameters derived from satellite sounders and an inversion technique, such as the Improved Initialization Inversion (3I) procedure, are combined with ISCCP data for understanding cloud radiative effects [Stubenrauch et al., 1996].

The climatic importance of cirrus clouds is well recognized [Liou, 1986; Slingo and Slingo, 1988], but their actual amount is still uncertain. Very little is known about multilayered cloud systems and, more generally, vertical cloud distribution. Yet the vertical distribution of clouds is important in determining the surface and top-of-atmosphere (TOA) radiative budgets. Outgoing longwave radiation is controlled by cloud top height, 
while downwelling longwave radiation depends on the cloud base. Moreover, the atmospheric and surface heating rates, which are the quantities that eventually affect the atmospheric motions, cannot be inferred from the surface and TOA radiation budgets but depend on the exact vertical structure of the atmosphere and cloudiness [Randall et al., 1989; Charlock et al., 1994]. Few studies report information on cloud vertical structure. Warren et al. [1985] reported statistics for the occurrence of simultaneous cloud types from surface observations. Baum et al. $[1994,1995]$ did a multilevel cloud analysis through the use of merged HIRS and AVHRR data. Sheu et al. [1997] combined infrared (AVHRR) and special sensor microwave imager information to retrieve cloud stratification in tropical areas. However, a much larger effort is needed in this direction, and new observational systems should be designed and tested. In light of previous sensitivity analyzes [Chou, 1985; Del Genio, 1992; Hansen, 1992; Zhang et al., 1995] it appears that approximate absolute accuracies of (1) a few tenths for cloud optical depth, (2) a few percent for cloud fraction as a function of cloud type or altitude, and (3) $200 \mathrm{~m}$ on cloud altitude retrieval are appropriate for new observational systems. The detection and quantification of horizontal cloud inhomogeneities and semitransparent cirrus clouds are also goals for the new cloud observational studies.

Backscatter lidar systems have been used for a long time to retrieve the structural and optical properties of cirrus clouds from the ground and aircraft [Platt, 1973; Spinhirne et al., 1982; Spinhirne and Hart, 1990]. Spaceborne observation has recently been emphasized by the Lidar In-space Technology Experiment (LITE) [McCormick et al., 1993; Winker et al., 1996]. The Earth Radiation Mission has been proposed by the European Space Agency (ESA) [ESA, 1996], with a payload that includes a backscatter lidar, scanning radiometers, and a cloud radar. The aim of the mission is to improve the retrieval of radiative parameters and cloud microphysics (water content and effective radius) through a combination of the various instruments.

Lidars and radars are complementary new instruments for cloud observation from space: lidars detect optically thin clouds [McCormick et al., 1993], while radars can penetrate dense clouds [Brown et al., 1995]. However, they require dedicated studies and technology demonstration. As far as lidar is concerned, the LITE mission proved the feasibility and capability of a spaceborne backscatter lidar [Winker et al., 1996], although it was limited to a short shuttle mission.

In this paper we address the potential contribution of a spaceborne backscatter lidar (SBL) to retrieve the vertical distribution of cloud fraction and optical depth. The emphasis is placed on the 3-D approach. Observed cloud data sets where multiple cloud layers are reported, such as the ground-based cloud climatology by Warren et al. [1985, 1986, 1988], could not be used for this study because full information on the vertical cloud structure, including the vertical distribution of cloud optical depth, is needed. Using an ad hoc (i.e., modeled) cloud climatology is actually the only way to circumvent the problem of not having any reliable 3-D cloud climatology. We thus used detailed results from a general circulation model (GCM) simulation, along with assumptions on the subgrid (i.e., not resolved) scale. The model cloudiness shows reasonable agreement with available data [Doutriaux et al., 1995a; Yu et al., 1996]. The cloud information retrieved from the lidar, and also from passive radiometers, is simulated from this GCM cloud data set. The two climatologies derived from the lidar and the radiometers are then compared and discussed. The paper is organized into five sections. The cloud data set obtained from the GCM is described in section 2. Section 3 presents our methodology to retrieve the 3-D cloud structure from the SBL and radiometers. The results are discussed in sections 4 and 5 .

\section{Cloud Data Set Used in the Study}

\subsection{Laboratoire de Météorologie Dynamique GCM}

As we discussed in the introduction, a cloud database is constructed from a 1 month GCM simulation. The Laboratoire de Météorologie Dynamique (LMD) GCM [Sadourny and Laval, 1984] was used for this purpose. The resolution considered here is 64 points evenly spaced in longitude and 50 points evenly spaced in sine of latitude. The size of the model grid varies from about $625 \times 225 \mathrm{~km}^{2}$ at the equator to about $400 \times 400 \mathrm{~km}^{2}$ at $60^{\circ}$ latitude. The model has 11 vertical levels prescribed through the $\sigma$ coordinate $\left(\sigma=p / p_{s}\right.$, where $p$ and $p_{s}$ are the atmospheric and surface pressures, respectively). The $\sigma$ levels and the corresponding approximate pressure and altitude levels are given in Table 1.

Cloud water content is predicted through the cloud water continuity equation [Le Treut and $L i, 1991$ ]. Precipitation is a sink of cloud water and is parameterized differently for ice and liquid water clouds. Cloud fraction is predicted by a simple statistical scheme [Le Treut and $L i, 1991$ ] at each of the vertical levels. On the vertical, clouds are assumed to fill the whole altitude range of a cloudy level. On the horizontal the cloud is defined by the cloud fraction, and homogeneous cloud microphysical and macrophysical properties are assumed. The radiative scheme is due to Fouquart and Bonnel [1980] for the SW part and Morcrette [1991] for the LW part. A random cloud overlap assumption is used to compute the radiative fluxes and heating rates throughout the integration of the model. There is no diurnal cycle in the version of the model used here [Doutriaux et al., 1995a]. This is not a limitation of the present study, because the Sun-synchronous satellite we consider here cannot sample the diurnal cycle. 
Table 1. Distribution of the Vertical Levels in the LMD GCM

\begin{tabular}{lccccccccccc}
\hline GCM Level & 1 & 2 & 3 & 4 & 5 & 6 & 7 & 8 & 9 & 10 & 11 \\
\hline$\sigma=p / p_{s}$ & 0.989 & 0.960 & 0.907 & 0.821 & 0.704 & 0.563 & 0.414 & 0.274 & 0.157 & 0.072 & 0.019 \\
Pressure, $\mathrm{hPa}$ & 1002 & 972 & 919 & 832 & 713 & 570 & 420 & 278 & 159 & 73 & 19 \\
Altitude, $\mathrm{m}$ & 90 & 340 & 820 & 1630 & 2870 & 4600 & 6800 & 9700 & 13200 & 17500 & 23500 \\
\hline
\end{tabular}

Approximate pressure and altitude of the sigma levels are computed assuming a standard atmosphere.

\subsection{GCM Cloud Database}

A four-dimensional (space and time) cloud database is built by runnning the LMD GCM for the month of July, with sea surface temperature conditions of July 1987. The relevant atmospheric parameters, such as temperature, humidity, cloud water content, and cloud fraction, are sampled every 3 hours on the totality of the 35,200 (i.e., $11 \times 64 \times 50$ ) grid points. The cloud distribution simulated by the LMD GCM has been previously compared with surface-based [Warren et al., 1986, 1988] and the ISCCP satellite-based [Rossow et al., 1991] cloud climatologies. At about $60 \%$ the overall cloud fraction is comparable to the observations of ISCCP (as the fraction of cloudy pixels) and surface stations (as the fraction of the sky dome covered by clouds) [Doutriaux et al., 1995a, b; Yu et al., 1996]. Following the methodology for cloud validation described by $Y u$ et al. [1996], we found midlevel clouds to be underestimated, whereas low level clouds (stratus) were overestimated in comparison with the ISCCP C1 cloud data. Stratocumulus clouds are missing off the west coasts of North America, Africa, and South America. To avoid contamination by spurious very thin model clouds, only clouds with visible optical depths larger than 0.1 are considered here. The simulated LW cloud radiative forcing, which is basically a signature of highlevel clouds, is in good agreement with the Earth Radiation Budget Experiment (ERBE) data in the tropics and midlatitudes but is overestimated at high latitudes [Bony et al., 1992]. Multilayered cloud situations are frequent; the (instantaneous) frequency distribution of the number of cloudy model levels is typically bimodal. It is apparent from Figure 1 that this distribution has modes at two to three and five to six cloudy layers. The number of cloudy levels simulated by the GCM is expected to be larger than the number of cloudy layers in the real world because contiguous cloudy levels may in fact pertain to a single cloud. While this may appear as a drawback of our study, we actually bound the problem by considering the maximum and random cloud overlap assumptions, as explained below.

\subsection{Cloud Distribution at the Subgrid Scale}

Observations of cloud overlap are scarce. Using U.S. Air Force Three-Dimensional Nephanalysis (3DNEPH) cloud analyses, Tian and Curry [1989] showed that cloud overlap is a function of spatial resolution. Mokhov et al. [1995] attempted to derive cloud overlap from observation; however, the reported value of cloud overlap depends not only on cloud type and geographical region, but also on the horizontal and vertical resolutions of the observing system or model. Two different assumptions, commonly used in GCM simulations, are considered in the present study: the random cloud overlap assumption and the maximum cloud overlap assumption [Charlock et al., 1994; Mokhov et al., 1995].

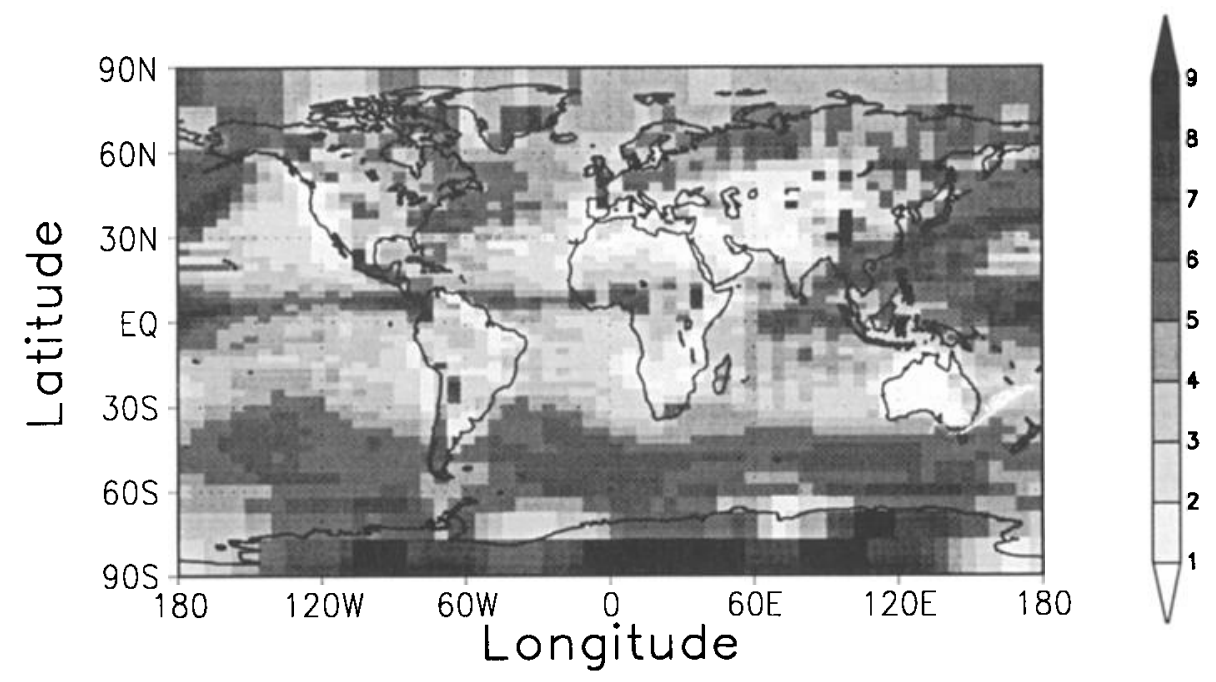

Figure 1. Average number of cloudy levels simulated by the general circulation model (GCM) of the Laboratoire de Météorologie Dynamique (LMD). 


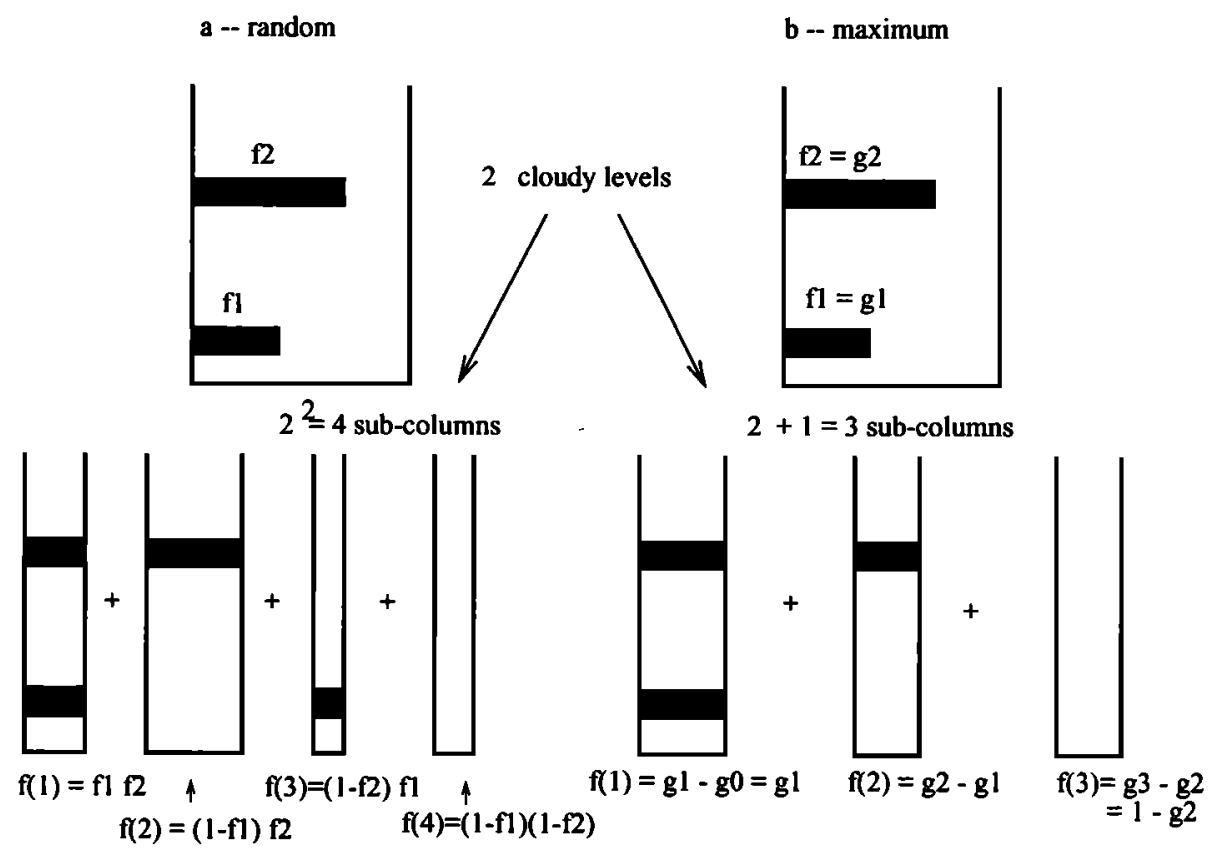

Figure 2. Diagram of the distributions of atmospheric subcolumns within a model grid column for (a) the random cloud overlap assumption and (b) the maximum cloud overlap assumption. See text for more details.

Because the objective here is to retrieve not only the total cloud cover but also its vertical distribution, we subsequently divide each GCM column into subcolumns of homogeneous horizontal properties according to the cloud overlap assumption.

2.3.1. Random cloud overlap assumption. The cloud fractions at the different levels are assumed to be independent random variables. We define $2^{l_{m}}$ subcolumns, where $l_{m}$ is the number of vertical cloudy levels in the model. Subcolumns are characterized by uniform horizontal properties (a level in a subcolumn is either totally cloudy or cloud free; see Figure 2a). The fractional area of a given subcolumn col is given by

$$
f(c o l)=\prod_{l=1}^{l_{\max }}\left\{i_{l}(c o l) f_{l}+\left[1-i_{l}(c o l)\right]\left(1-f_{l}\right)\right\}
$$

where $l_{\max }$ is the number of model levels and $f_{l}$ is the cloud fraction at level $l$. The cloud presence indicator, $i_{l}(c o l)$, is 1 if level $l$ is cloudy; otherwise it is 0 .

2.3.2. Maximum cloud overlap assumption. Cloud overlap is assumed to be maximum on the vertical. This situation is particularly representative of deep convection at tropical latitudes. The number of subcolumns is equal to the number of model cloudy levels, $l_{m}$, plus one for clear sky (Figure 2b). The fractional area of subcolumn is given by:

$$
f(c o l)=g_{\text {col }}-g_{c o l-1} \text { with } c o l=1 \text { to } l_{m}+1
$$

where $g_{l}$ are the $l_{m}$ cloud fractions in ascending order, $g_{0}=0$ and $g_{l_{m}+1}=1$ (see Figure 2b).

These two cloud overlap assumptions lead to two different frequency distributions of the subcolumn areas.
The average area of individual subcolumns is smaller for the random cloud overlap assumption, which produces a larger number of subcolumns (see Figure 1). By decreasing the average area of the subcolumns the random cloud overlap is a way to represent some degree of inhomogeneity at the mesoscale. Subcolumn areas are correspondingly larger for the maximum cloud overlap assumption. Low level clouds are more readily overlapped by upper level clouds with the maximum cloud overlap assumption than they are with the random cloud overlap assumption (compare $f(1)=f_{1} f_{2}$ and $f(1)=g_{1}-g_{0}$ in Figure 2). Therefore we anticipate that it will be easier to detect low level clouds under the random cloud overlap assumption. Also, the total cloud cover is higher with the random cloud overlap assumption.

\section{Methodologies for the Retrieval of Cloudiness}

We now describe the detection schemes for the observing systems we simulate, keeping in mind that the objective is to retrieve the total cloud cover and its vertical distribution. The lidar and radiometer cloud detection schemes are applied to the subcolumns of each pixel (or grid box) overpassed by the satellite. The satellite is assumed to be on a polar orbit, at an altitude of about $800 \mathrm{~km}$. The analysis procedure is divided into four steps:

1. The GCM grid boxes are sampled according to the satellite orbit and divided into independent subcolumns using the two selected cloud overlap assumptions.

2. Each subcolumn is tested which cloud layers are 
Table 2. Lidar System Parameters Considered in the Present Study

\begin{tabular}{ll}
\hline \multicolumn{1}{c}{ Parameter } & \multicolumn{1}{c}{ Value } \\
\hline Transmitted energy, mJ & 100 \\
Wavelength, $\mu \mathrm{m}$ & 1.064 \\
Repetition rate, $\mathrm{Hz}$ & 100 \\
Telescope diameter, cm & 60 \\
Overall efficiency $^{*}$ & 0.15 \\
Swath, km & 600 \\
Orbit altitude, $\mathrm{km}$ & 800 \\
\hline \multicolumn{2}{c}{ Optical transmission and detector } \\
quantum yield.
\end{tabular}

detected by the SBL. This point is further discussed in section 3.1 .

3. An ISCCP-like algorithm is applied to individual subcolumns to derive the cloud top pressure of an equivalent single cloud layer producing the same infrared radiative flux at the TOA. The cloud optical depths are used to correct the cloud top pressure as would be done in the ISCCP algorithm from the information contained in the visible (VIS) channel. This step is discussed in section 3.2 and further described by Yu et al. [1996].

4. The SBL- and radiometer-retrieved global cloud distributions are reconstructed from the sampled grid boxes and compared with the initial model cloud distribution.

Steps 2,3 , and 4 are conducted under the two cloud overlap assumptions.

\subsection{Lidar}

The pulsed backscatter lidar provides range-resolved information on the scattering properties of the atmosphere. The backscattered signal is enhanced in the presence of clouds. It is also attenuated as the beam propagates through clouds. This effect results in a signal peak that can be used to define the occurrence of a cloud and its range. The cloud detection capability of a backscatter lidar thus depends on the signal-to-noise ratio (SNR), which can be derived at cloud top from the system characteristics. Assuming background noise limitation, the SNR can be written as

$$
\mathrm{SNR}=C \alpha \sqrt{N} T^{2}
$$

where $C$ is a constant (with dimension of length) depending on the lidar characteristics (transmission and detection efficiency, telescope size, emitted energy, squared range) and cloud properties (backscatter to extinction ratio), $\alpha$ is the cloud extinction coefficient, and the atmospheric transmission can be expressed as $T=\exp \left(-\tau_{u l}\right)$ with $\tau_{u l}$ being the optical depth of the upper layers. Calculations of SNR using lidar parameters have been performed as reported in Table 2. These parameters are representative of the atmospheric lidar (ATLID) spaceborne system as proposed by ESA [ESA, 1996]. Using such parameters, the background limited
SNR equation only applies to optically thin cirrus and multilayered clouds. The use of such a formulation is thus detrimental to the detection of a dense cloud in a single layer. However, it is used for the sake of simplicity, as this last case was not frequently simulated (see cloud layer statistics given in Figure 1). A single value of the backscatter to extinction ratio $\left(0.05 \mathrm{sr}^{-1}\right)$ has been assumed in the simulations for liquid and ice clouds; this yields a value of $C$ equal to $1.3 \mathrm{~km}$. An SNR value of 4 allows one to extract the cloud top signal from the noise with a false alarm probability below $5 \%$, over an altitude domain corresponding to a possible cloud occurrence [Pelon et al., 1996]. Such an SNR value requires about 100 shots (10 shots) to detect a cloud with extinction coefficient of $0.3 \mathrm{~km}^{-1}\left(1 \mathrm{~km}^{-1}\right)$. Denser clouds, with extinction coefficient greater than $3 \mathrm{~km}^{-1}$, are detected in a single shot in the absence of upper level clouds. In the presence of a multilayered cloud structure the attenuation by upper level clouds would be the limiting factor, due to the effective dynamical range of the system. We do not account for a limited response time for dense cloud detection. This is not expected to introduce significant bias because the transmission through dense clouds rapidly decreases at lower levels. Multiple scattering effects leading to a decrease in the attenuation [Platt, 1979] have not been considered either. However, they can lead to a significant improvement in low cloud detection, as was demonstrated in the LITE experiment [Winker et al., 1996].

The scanning capability of the lidar systems under consideration results in a homogeneous distribution of shots over a full swath of $600 \mathrm{~km}$, using a $100 \mathrm{~Hz}$ repetition rate laser [Pelon et al., 1996]. This leads to a regular shot spacing of about $6 \mathrm{~km}$. For the GCM the number of shots is proportional to the size of the grid box (e.g., about 7000 shots per grid box near the equator). Note that the swath of the lidar is used to predict the spatial distribution of lidar shots per surface unit. It is not used to predict which portion of the GCM grid box area is actually sampled. This approach would make little sense, since the cloud distribution at the subgrid scale is statistical.

At this stage we considered two lidar schemes: a first one corresponding to a detection capability limited by the SNR as previously defined and a second one corresponding to a detection capability limited only by the attenuation. The cloud detection performance of this first lidar is related to the SNR value computed over each subcolumn from the number of shots, transmission, and cloud extinction coefficient. The number of shots, $N$, reaching a given subcolumn assumes a uniform laser shot density over the model grid box and is the product of subcolumn fraction area and total number of shots over the model grid box. The cloud detectability is therefore a function of the distribution of subcolumn areas and, in turn, the cloud overlap assumption. The cloud extinction coefficient $\alpha$ is computed as the ratio of the cloud optical depth to the model level thickness. 


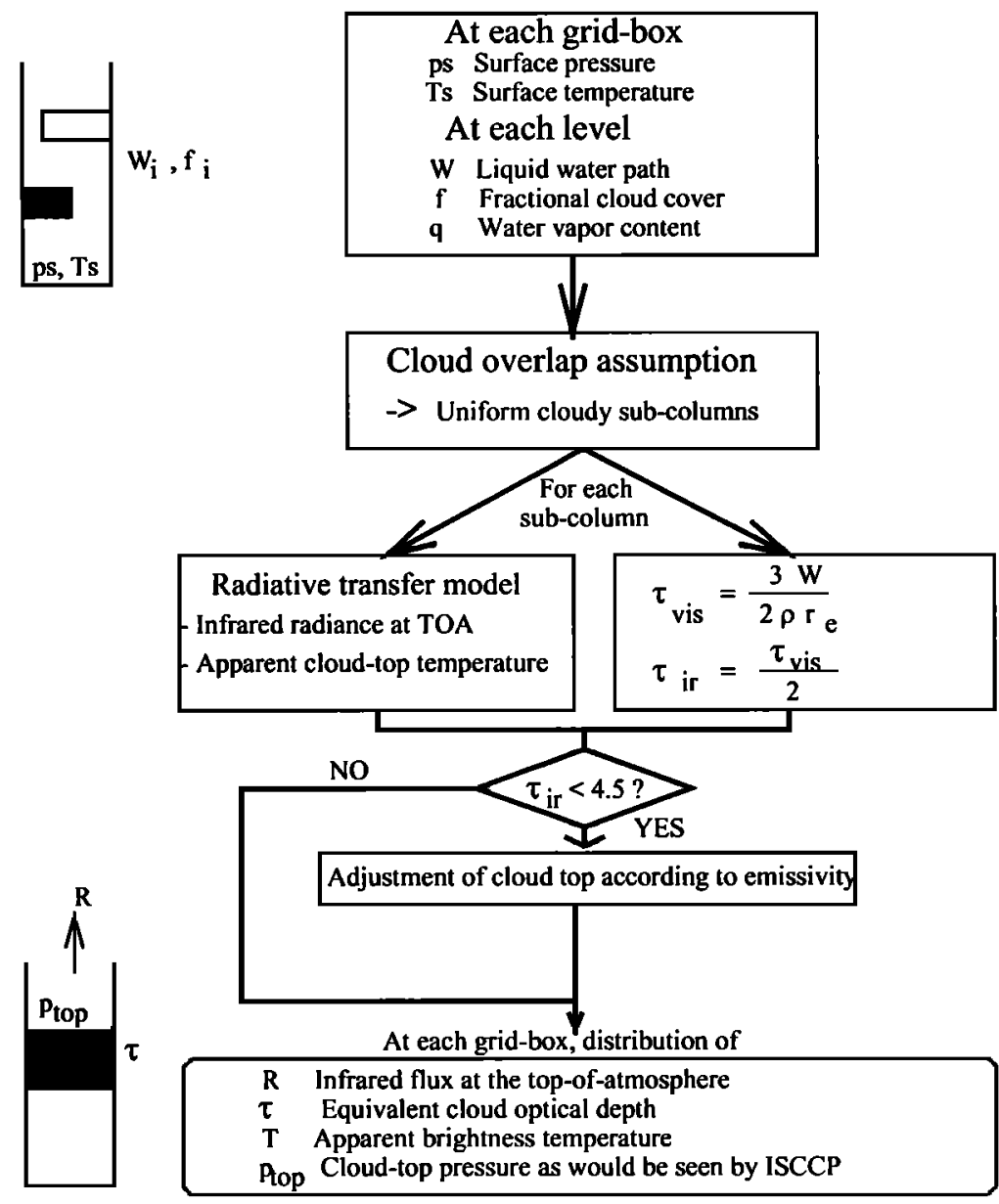

Figure 3. Schematic diagram of the ISCCP-like algorithm, also referred to as the IR+VIS channel scheme. Here, $W$ is the vertically integrated water content, $r_{e}$ is the effective cloud droplet radius, $\rho$ is the water density, and $\tau_{\text {vis }}$ and $\tau_{\text {ir }}$ are the visible and infrared cloud optical depths, respectively.

This first scheme is referred to as an "SNR-limited lidar." The detection performance in the second scheme is independent of the cloud backscatter, cloud extinction coefficient, or number of shots. This corresponds typically to a system working at high SNR, allowing detection from a single shot, as long as the optical depth threshold is not reached [Pelon et al., 1996]. This lidar will be referred as an "ideal lidar." On the basis of signal dynamics range of typical existing backscatter lidars [Spinhirne and Hart, 1990] we have set the attenuation threshold to correspond to an integrated optical depth of 3 between the lidar and the cloud layer top. Above this value no cloud is detected. For both lidar systems the cloud layer fraction retrieved at one model level is the sum of the cloudy subcolumn areas detected at that level.

\subsection{Radiometers}

We also simulate the radiometer cloud retrieval, following a procedure described in detail by $Y u$ et al. [1996]. This procedure is close to the ISCCP cloud re- trieval algorithm [Rossow et al., 1991]. It is assumed here that the radiometers detect all the cloudy subcolumns and their respective areas. The key point is therefore the detection of the altitude of an equivalent cloudy layer. As for the ISCCP algorithm, and whatever the number of cloudy levels is, a single layer of cloudiness can be retrieved. The altitude of the equivalent cloudy layer is estimated for each cloudy subcolumn in four successive steps. First, we calculate, for each subcolumn, an upward infrared (IR) radiance at the TOA, from the model temperature, water vapor, and cloud vertical profiles. This corresponds to the radiance that would be measured by a nadir-looking satellite. Second, TOA IR radiances are computed by, using the same temperature and water vapor profiles and assuming a single-layer, IR radiation opaque cloud, which is placed successively at each of the model levels. The model level giving the best fit to the TOA IR radiance computed in the first step is the level of the equivalent cloud retrieved by the radiometry. Third, the cloud optical depth $\tau_{\text {VIS }}$ is computed as the sum 
Table 3. Monthly Mean Model Cloud Fractions and Sampled Cloud Fractions From the Polar-Orbiting Satellite

\begin{tabular}{cccc}
\hline Model Level & \multicolumn{2}{c}{ Cloud Fraction, \% } & Relative Difference, \% \\
\cline { 2 - 3 } & All Grid Boxes & Sampled Grid Boxes & \\
\hline 1 & 8.8 & 8.9 & 1.6 \\
2 & 17.3 & 17.4 & 0.3 \\
3 & 26.0 & 26.0 & 0.0 \\
4 & 13.0 & 13.0 & 0.1 \\
5 & 6.2 & 6.3 & 0.3 \\
6 & 8.4 & 8.4 & 0.1 \\
7 & 11.8 & 11.7 & 0.2 \\
8 & 17.6 & 17.5 & 0.4 \\
9 & 20.6 & 20.6 & 0.1 \\
10 & 0.8 & 0.7 & 2.6 \\
11 & 0.0 & 0.0 & 0.0 \\
\hline
\end{tabular}

The relative difference between the two (in percent) is indicated in the last column.

of the model cloud optical depths over the vertical of the subcolumn, assuming cloud droplets with effective radius of $10 \mu \mathrm{m}$. Fourth, $\tau_{\mathrm{VIS}}$ is used, if necessary, to adjust the cloud top pressure (and the corresponding model level) determined during the second step. Since most optically thin clouds are likely to be cirrus, an empirical relation between the infrared optical depth $\tau_{\mathrm{R}}$ and the visible optical depth $\tau_{\mathrm{VIS}}$ is assumed where $\tau_{\mathrm{IR}}=0.5 \tau_{\mathrm{VIS}}$, following Platt and Stephens [1980] and Rossow et al. [1991]. If $\tau_{I R}$ is greater than 4.5 (corresponding to a transmission less than $1 \%$ ), the cloud is considered opaque, and no adjustment is done. If not, the cloud is considered semitransparent, and the cloud top temperature is adjusted accordingly. A flowchart of this retrieval procedure is given in Figure 3. This retrieval scheme is referred to as the "IR+VIS channel" scheme. At night, only the first two steps can be achieved, and the scheme is referred to as the "IR channel only" scheme. Since there is no diurnal cycle in the GCM, the IR channel only scheme and the IR+VIS channel schemes are applied to the whole data set.

In ISCCP a cloudy pixel is assumed to be completely and uniformly covered by clouds. Although this assumption can bring errors in the real world ISCCPderived cloud cover and cloud optical properties, it is not the case here, as we are working directly on homogeneous subcolumns rather than pixels.

\section{Results}

\subsection{Effect of Sampling From a Polar-Orbiting Satellite}

Whereas the ISCCP cloud data set is obtained from a large number of observations from both geostationary and polar-orbiting satellites, a single polar-orbiting satellite achieves a rather low sampling of the Earth's cloudiness. Although it is beyond the scope of this study to discuss the systematic and random errors caused by sampling from a polar-orbiting satellite, we first examine this point with our GCM cloud data set. A similar approach was used by Barkstrom [1997] to simulate the orbital coverage of the geoscience laser altimeter system and the Pathfinder instrument for cloud and aerosol spaceborne observations satellites in the Colorado State University GCM. We compute the cloud fraction of the model from the polar-orbiting satellite sampling (25 samples per grid box and per month) and compare it with the initial field, which includes all the samples ( 240 samples per grid box and per month). The instrument has a transverse scanning with a full swath of the size of a model grid box at the equator (see Table 2). For now we assume that the cloud fraction is perfectly retrieved at all optical depths we discuss the performance of the detection schemes in the next sections. The number of observations in a sampled grid box is assumed to be large enough to introduce no additional limitation. Since the GCM we are using has no diurnal cycle, it is not possible to assess the systematic errors arising from the diurnal bias of such a polar-orbiting satellite. Rossow [1994] has shown that these errors are typically of the order of $0-15 \%$ for a nadir-sampled observation compared with an eight-times-daily ISCCP observation on a monthly mean average and a $2.5^{\circ}$ resolution. In contrast, it is possible here to assess the random errors due to undersampling. Results are listed in Table 3 as global averages at different model levels. It is seen that the differences between the full- and the satellite-sampled observations remain below $0.6 \%$ except for GCM levels 1 and 10. This finding means that a single satellite carrying an ideal SBL with a swath of about $600 \mathrm{~km}$ is sufficient to retrieve an accurate global monthly mean cloud fraction. Because the temporal variability of the low level cloud cover is small in our GCM, the impact of undersampling remains small in the lower troposphere (Figure $4 \mathrm{a}$ ) but increases at higher levels (Figure $4 \mathrm{~b}$ ), where it can be significant in some regions. 


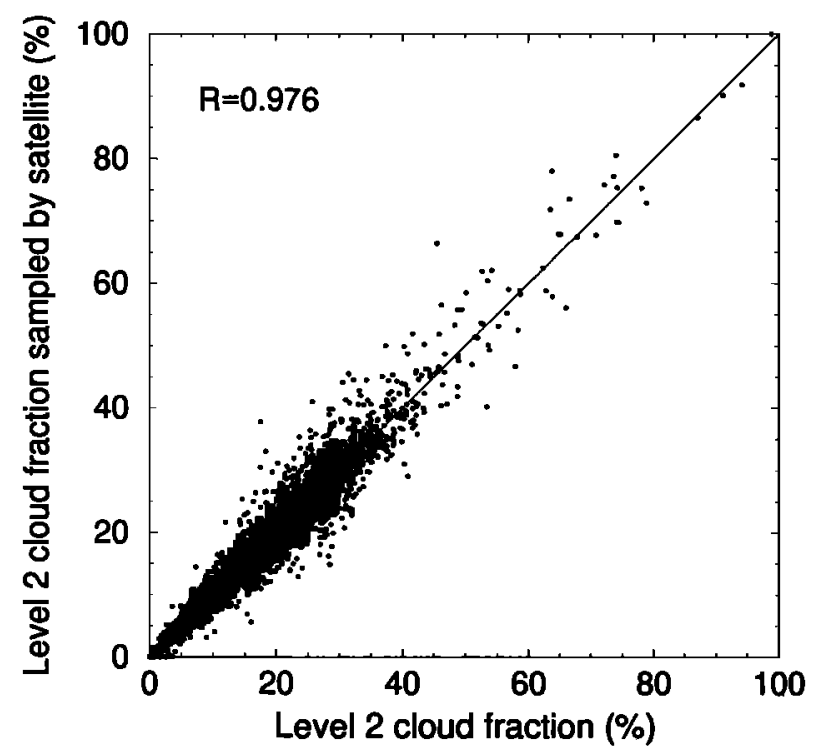

(a)

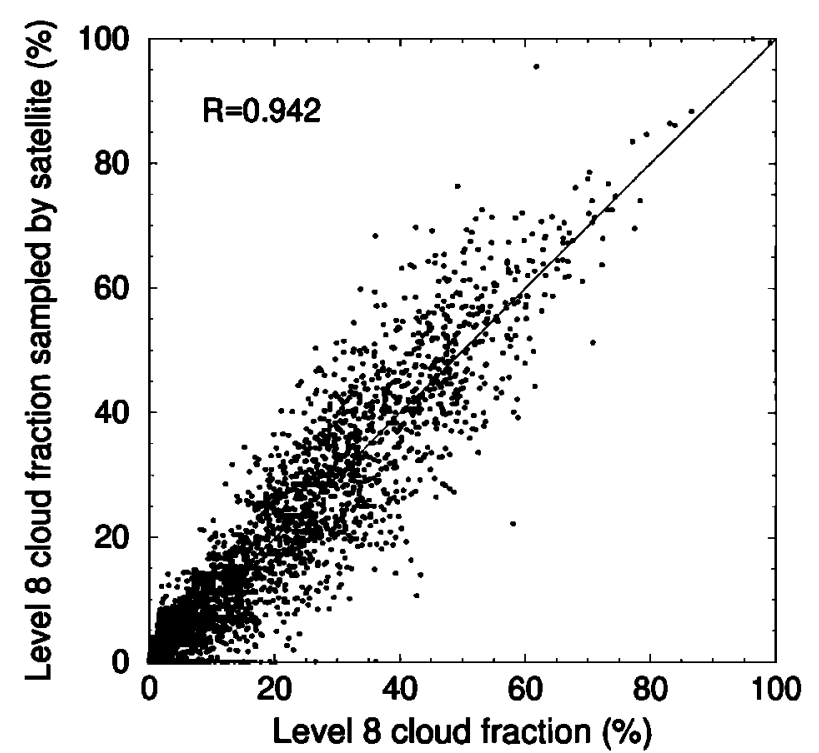

(b)

Figure 4. Scatterplots of the sampled versus the original cloud fraction on a model grid box basis for (a) low-level clouds (model level 2 at about $340 \mathrm{~m}$ ) and (b) high-level clouds (model level 8 at about $9700 \mathrm{~m}$ ).

\subsection{Lidar Cloud Retrievals}

The vertical distribution of the cloud fractions retrieved by the simulated lidars and radiometers are shown in Figure 5 as global monthly averages for the two cloud overlap assumptions. The SNR limitation introduces significant differences for the detection of upper cloud layers. An ideal system detects about $100 \%$ of upper level clouds (e.g., model levels 8 and 9 at average altitudes of 9.8 and $13.6 \mathrm{~km}$ ) in contrast to $84 \%$ for an SNR-limited lidar using the maximum cloud overlap assumption. This limitation is due to the presence of both thin and dense clouds at high altitudes (Plate 1). Thin high level clouds, with average optical depth lower than unity, are found at middle and high latitudes. They are not detected if the number of shots is not sufficient and the SNR is below the threshold. Dense high level clouds occur in the tropics at the top of cloud turrets related to deep convection and may extend over large areas, as suggested by the larger average optical depths shown in Plate 1. In such situations the cloud attenuation dramatically increases in the lower layers, and underlying dense clouds cannot be detected. The maximum and the random cloud overlap assumptions give comparable results for high clouds, with a slightly better detection for the former assumption. Low level clouds are better detected under the random cloud overlap assumption, which may be more relevant in midlatitude regions. The two lidar detection schemes give similar results for low level clouds because the SNR is higher and somewhat counterbalances for transmission losses due to the presence of upper level clouds. A maximum of $61 \%$ of the model cloud fraction is retrieved at model level $3(700 \mathrm{~m})$ for the random cloud overlap assumption. The limitation in SNR does not introduce a major loss in the lidar detection performance in comparison with an ideal system. It is, however, critical for the detection of thin cirrus clouds.

\subsection{Passive Radiometer Cloud Retrievals}

The radiometer-retrieved cloud fractions are shown in Figure 5 as global averages for the two cloud overlap assumptions and for the two retrieval schemes (IR channel only and IR+VIS channels). As we discussed previously, adjustment from the VIS channel information improves the retrieval of upper level clouds. Both schemes (IR channel only and IR+VIS channels) give comparable results for low clouds, as optical depths are large and no correction is needed. As for the lidar, better results are obtained by using the random cloud overlap assumption. Under the maximum cloud overlap assumption the retrieved cloud fraction at levels 9 and 10 is about $74 \%$ of the model cloud fraction for an IR+VIS radiometer. If the IR channel only scheme is used, only $34 \%$ of the model cloud fraction is retrieved. The remaining cloud fraction is assigned to lower levels. In any case, there is a tendency to underestimate cloud top height. The IR channel only scheme shows better performances for midlevel layers. However, an analysis in terms of zonal averages reveals that the cloud fraction retrieved by radiometers can be larger than the original model cloud fraction for midlevel layers of subtropical latitudes (Figure 6d). Overestimation is clearly apparent on a grid box basis, as Plate 2 shows. This is 


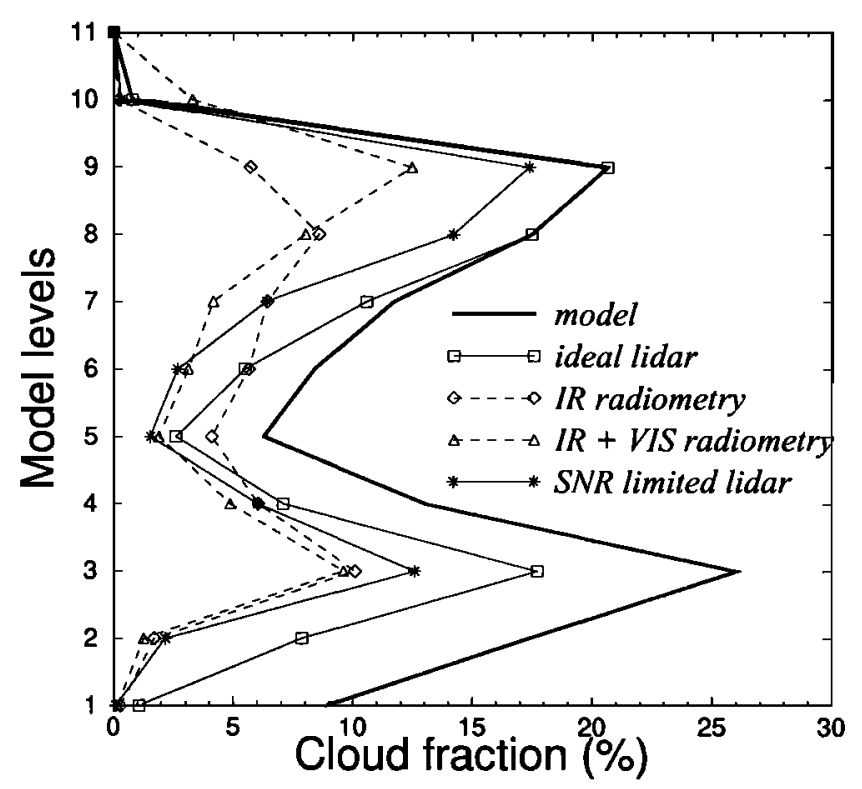

(a)

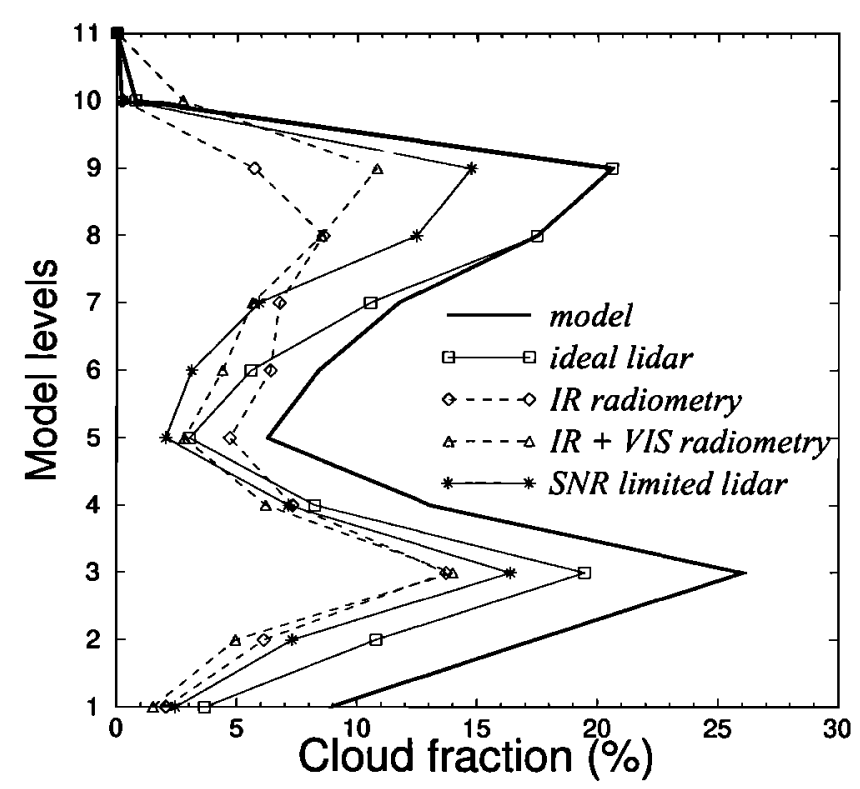

(b)

Figure 5. Comparison of lidar- and radiometer-retrieved cloud fractions with the model cloud fraction on a global average and according to the satellite sampling: (a) maximum cloud overlap assumption and (b) random cloud overlap assumption.

mostly the signature of low level clouds overlapped by high level clouds, which are retrieved as midlevel clouds.

\section{Comparison of Performances}

\subsection{High Level Clouds}

According to our analysis, a lidar would allow better high level cloud detection capabilities than would radiometers (IR+VIS channels), as Figure 6 shows. High level cloud detection and height assignment (i.e., at model levels 8, 9, and 10) are dramatically improved. However, significant differences are observed in upper level cloud detection between an ideal and an SNRlimited lidar. Cirrus clouds with optical depths larger than 0.1 are detected with a $100 \%$ probability in the former case and $84 \%$ in the latter case when the maximum cloud overlap assumption is used. This probability decreases to $71 \%$ for the random cloud overlap assumption, in comparison with $60 \%$ and $53 \%$ for the IR+VIS and IR radiometers, respectively. The differences in the results obtained under the two cloud overlap assumptions can be explained by the differences in subcolumn areas. For the random cloud overlap assumption, subcolumn areas are smaller, with a subsequent lower number of shots per subcolumn and a lower SNR value. For the passive radiometers the systematic underestimation is explained by the high occurrence of multilayered high clouds, which are retrieved at only one level. Plate 3 shows the difference in cloud fraction simulated by the GCM and retrieved by the lidar and radiometer systems at model level 9 (altitude, $\sim 13,200 \mathrm{~m}$ ) by using the random cloud overlap assumption. Both systems capture the spatial variability in high level clouds. The lidar appears to perform better and gives a better quantitative retrieval, although introduction of a realistic SNR limits the detection performance. The use of the IR channel only scheme leads to very poor results.

By simulating the lidar retrieval on equivalent homogeneous subcolumns, the lidar performs probably better than it would in the actual atmosphere, as thin cirrus clouds are often patchy and inhomogeneous at the mesoscale. The results also appear consistent with the fact that a single cloud layer is reported in each cloudy subcolumn in the radiometer retrieval. This leads to a lower retrieval by the radiometer of cirrus clouds embedded in large multilayered systems. It is conceivable that our procedure and the model cloud distribution enhance this detrimental effect. Although the radiometer performances depend on the original cloud fraction and analysis procedure, we note that the retrieved cirrus cloud fraction in our ISCCP-like simulation is $10-13 \%$, close to the ISCCP estimate [Yu et al., 1996]. This finding would support the idea, expressed in other places, that the ISCCP algorithm underestimates cirrus cloud cover.

\subsection{Midlevel Clouds}

For midlevel clouds (model levels 5,6 , and 7 ) the IR channel only radiometer reports the largest cloud fraction, while the lidar and IR+VIS radiometer exhibit comparable performances with a detection efficiency of $30 \%$ (see Figure 5). With the use of an SNR-limited lidar, midlevel clouds are even more poorly detected. Looking at zonal means, we again attribute this underestimation to the presence of thin clouds at high latitudes. However, if a high level cloud overlies a low level 


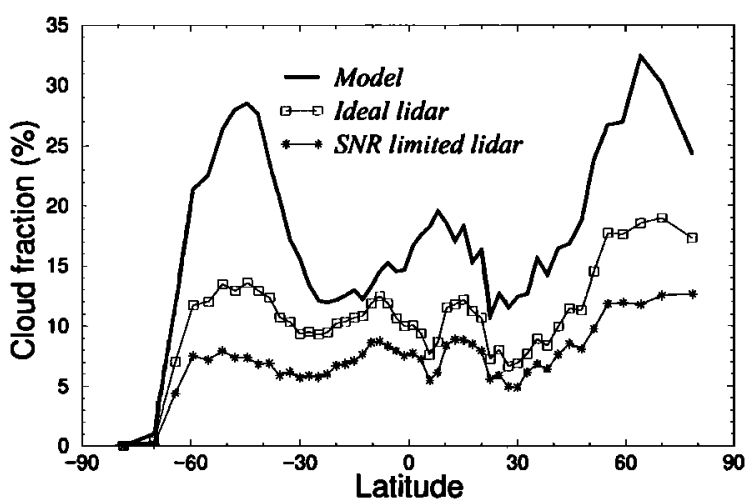

(a) Level 2 ( 340 m) - Lidar - Random



(c) Level 5 ( $2870 \mathrm{~m})$ - Lidar - Random



(e) Level 9 ( 13200 m) - Lıdar - Random

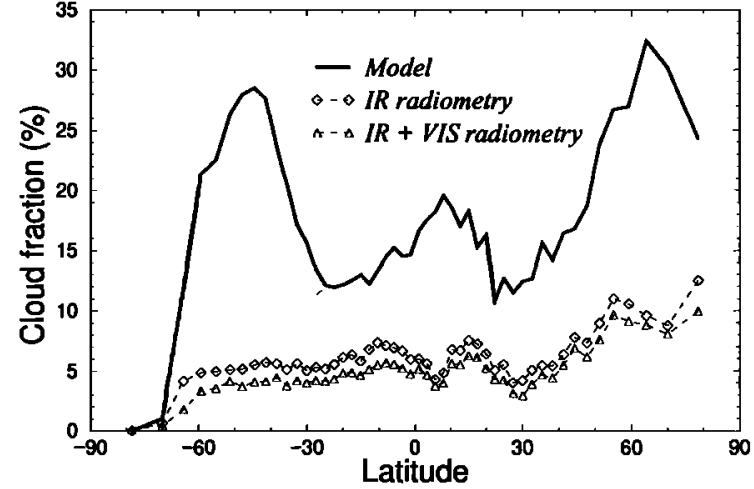

(b) Level 2 ( $340 \mathrm{~m})$ - Radiometry - Random

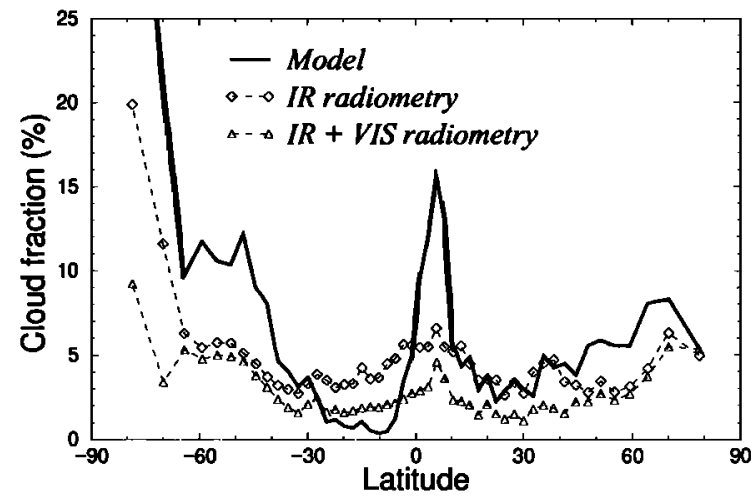

(d) Level 5 ( $2870 \mathrm{~m}$ ) - Radiometry - Random

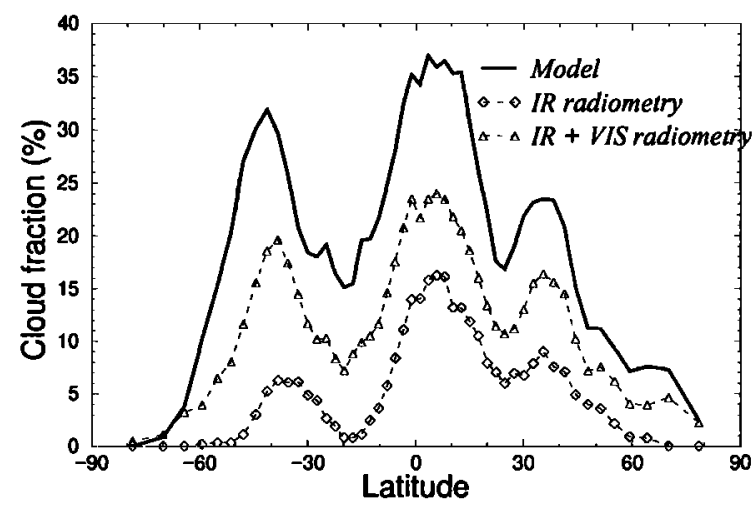

(f) Level 9 ( $\sim 13200 \mathrm{~m})$ - Radiometry - Random

Figure 6. Zonal mean cloud fraction retrieved by (a, c, e) lidar and (b, d, f) radiometry for different model levels and for the random cloud overlap assumption.

cloud, the IR+VIS radiometer often detects the cloud as midlevel. Therefore most of the midlevel clouds retrieved by the radiometer in this simulation correspond to multilayered clouds in the model. This finding is evidenced in Figure 6d, where the retrieved cloud fraction is higher than the model cloud fraction at tropical latitudes, mostly in the southern hemisphere. Comparisons between the retrieved and the model cloud fractions over individual grid boxes (Plate 2) also show that the passive radiometer retrievals can lead to much higher values, especially when the actual cloud fraction is small. An excess cloudiness of about $5 \%$ is observed on average.

\subsection{Low Level Clouds}

For low level clouds the lidar performance is degraded, but still better than that for the passive (IR + VIS) radiometer, with $50 \%$ and $40 \%$ retrieved cloud 


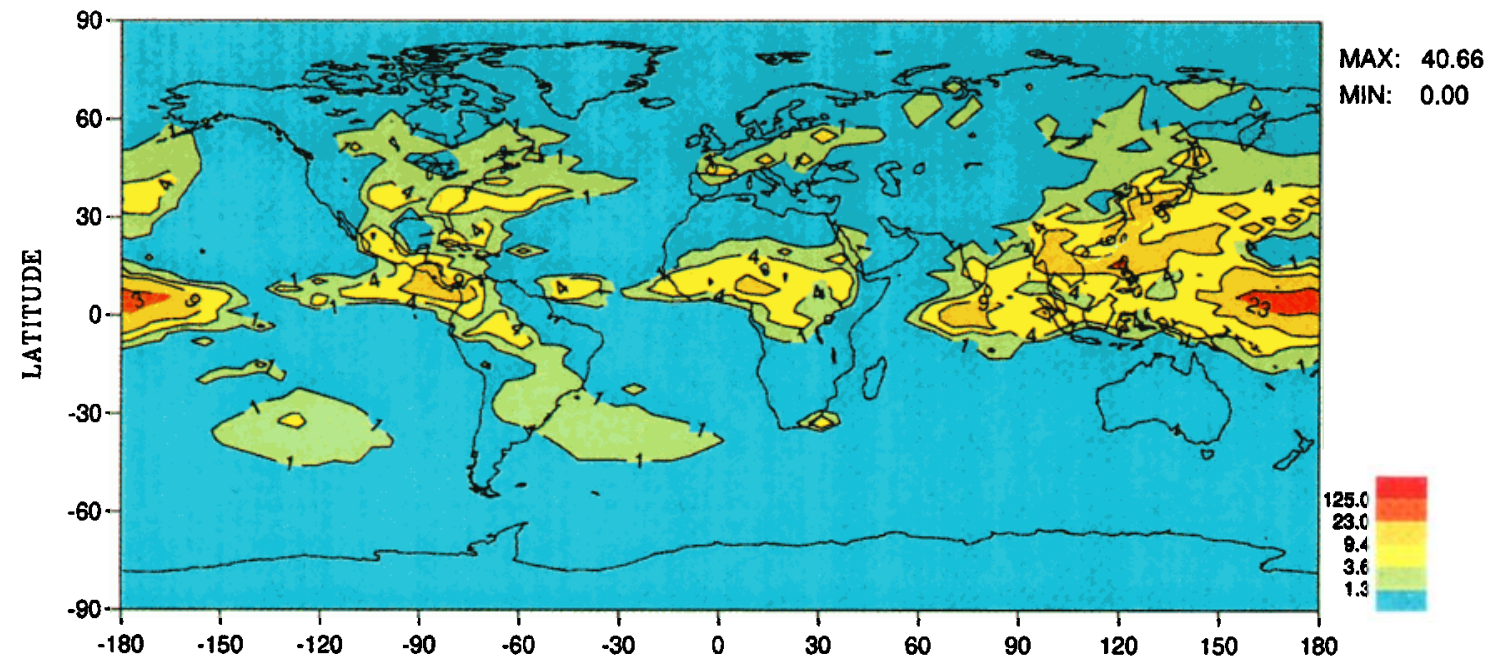

Plate 1. Cloud optical depth at model level $8(\sim 9700 \mathrm{~m})$.

fractions, respectively. This difference is mainly due to situations with simultaneous occurrences of a low level cloud and a thin cirrus. In such a situation the lidar can detect a low level cloud and reports its exact altitude, while the radiometer gives an equivalent cloud level between the two cloud levels. The attenuation by upper cloudy layers makes it difficult for the lidar to detect low level clouds when two or more dense cloud layers are present. However, the detection of a multilayered cloud system should further benefit from the cloud inhomogeneities at the mesoscale, as the lidar spot size is only a few hundred meters. We also assumed permanent daytime measurements, which allow the use of a correction scheme for the altitude assignment of the radiometer retrieval but reduce the detection capability of the lidar. Better lidar performance would be obtained during nighttime operation, when radiometry is limited to the IR channel detection only. Therefore both instruments appear complementary: cloud fraction, cloud layer altitudes, and the optical depth of semitransparent clouds could be accurately retrieved. This is an advantage of combining lidar and radiometer measurements, as the optically thin high level clouds important to the Earth's radiative budget are very poorly detected during nighttime.

\subsection{Sensitivity to Lidar Characteristics}

The performance of an SNR-limited lidar needs to be improved. Because the cloud detectability is limited by the cloudy subcolumn areas, as discussed in section 3.1 , there is a need for further improving the lidar detection performance of optically thin cirrus clouds over small cloudy areas during daytime. It is possible to decrease the swath to about $300 \mathrm{~km}$ and shift the satellite to a lower orbit $(400-600 \mathrm{~km})$ to increase both the spatial density of shots (by a factor of 2) and the SNR (by a factor of close to 3). Improvements in the algorithms that would allow cloud detection at SNR as low as 2 with a small false alarm error are also under study [Flesia et al., 1996].

The use of a nonscanning system has the advantage of a simplified design. It leads to an improved horizontal resolution and to substantial improvements in the SNR, but this configuration is detrimental to the horizontal representativeness of the measurements. Combining a nonscanning lidar and high-resolution IR+VIS imagers on a same platform may thus be considered as a compromise. Such a solution is under study at ESA in the framework of the Earth Radiation Mission.

\section{Conclusion}

The detection of multilayered cloud systems and proper retrieval of the cloud altitude are critical to determine the Earth-atmosphere radiative budget. A key problem for radiometers is to retrieve cloud boundaries (especially during nighttime) and to detect multilayered cloud systems. A spaceborne backscatter lidar, used in synergism with a radiometer, could overcome some of these limitations.

This study provides a first assessment of how well cloud fraction and cloud vertical structure are retrieved by a spaceborne backscatter lidar using a three-dimensional GCM cloud distribution. Simulations were also performed for an IR channel only and a two-channel (IR+VIS) spaceborne radiometer, using an ISCCP-like analysis scheme. This is so far the only way to conduct a meaningful comparison of cloud detection performances between several instruments. Further improvements including more representative spatial cloud features, such as cloud inhomogeneities, should be considered. This study could also be repeated with another GCM cloud data set or when a more comprehensive cloud data set becomes available. Our main results are stated below.

Random errors caused by sampling of a polar-orbiting satellite are small on a monthly average even for a re- 


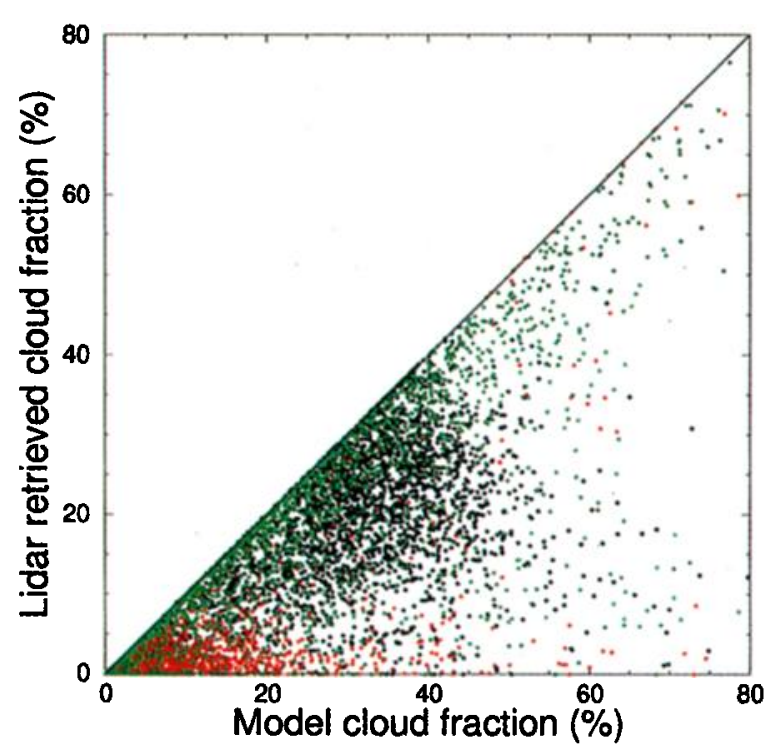

(a)

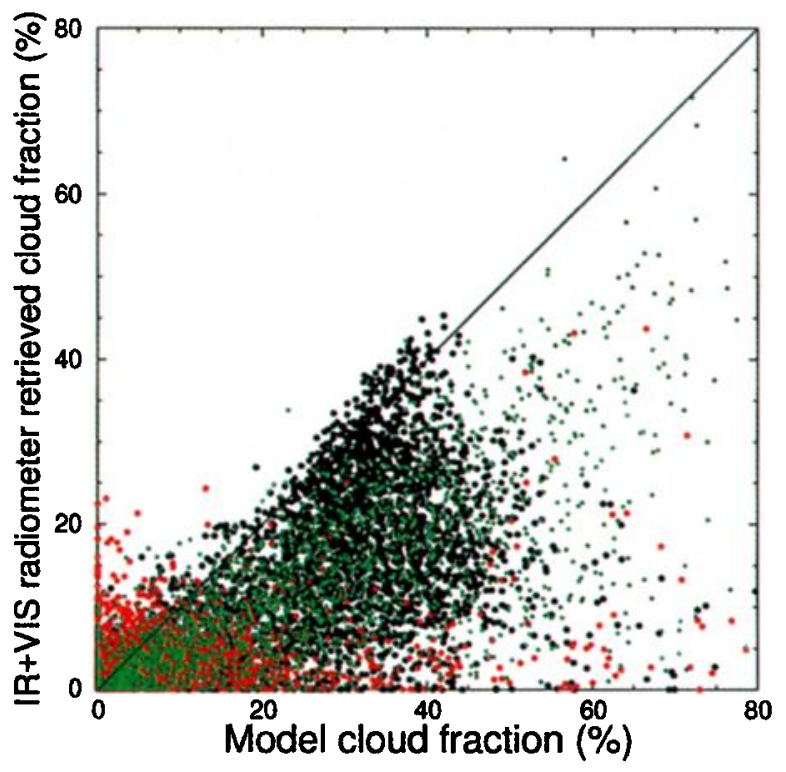

(b)

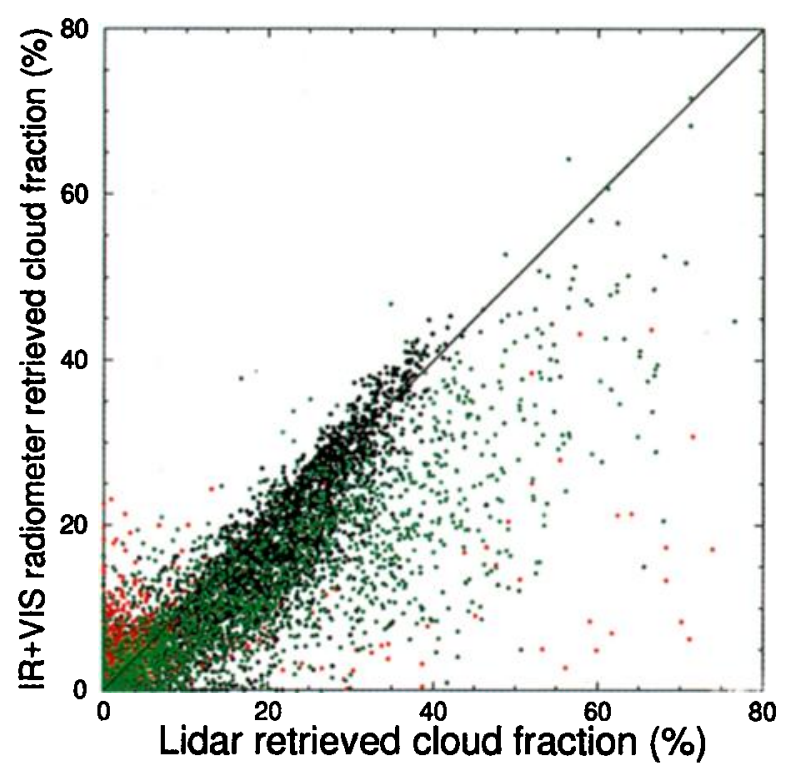

(c)

Plate 2. Scatterplots of lidar-retrived cloud fractions, radiometer-retrieved cloud fractions, and model cloud fractions on a grid box basis using the random cloud overlap assumption. Black dots, model level 3; red dots, model level 5; and green dots, model level 8. The retrieved cloud fractions in Plate $2 \mathrm{~b}$, which are above the 1:1 line, are an illustration of the limitations of the radiometry inversion in the presence of multilayered cloud structures. 


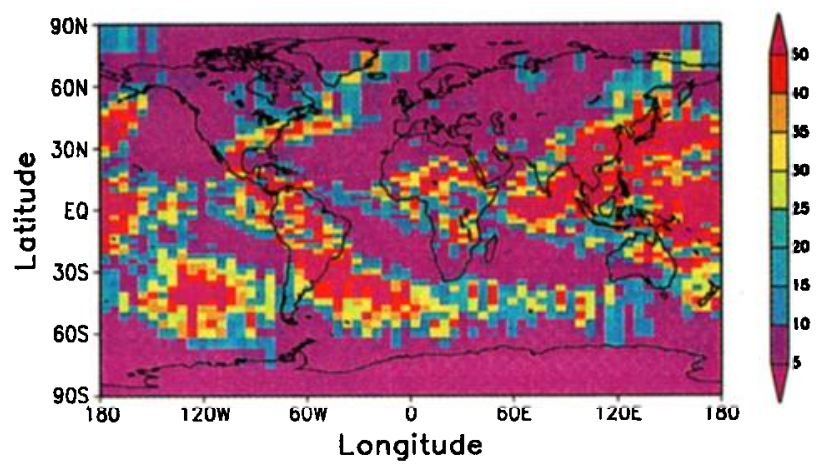

(a) Model

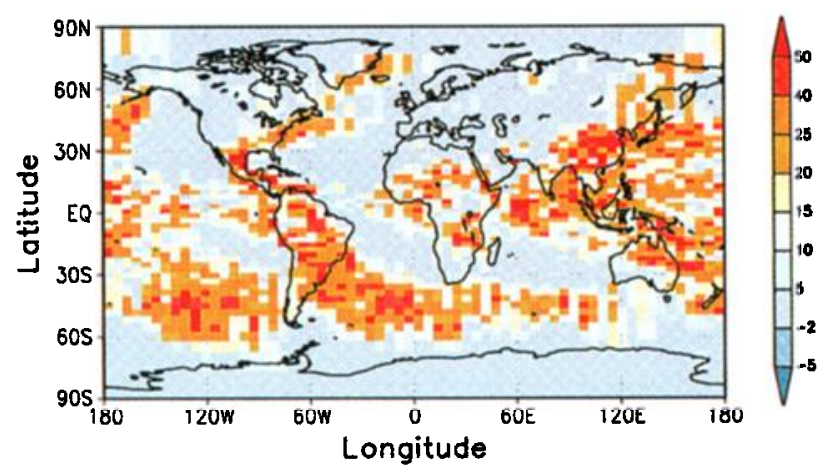

(c) Model - Radiometry, IR channel only scheme

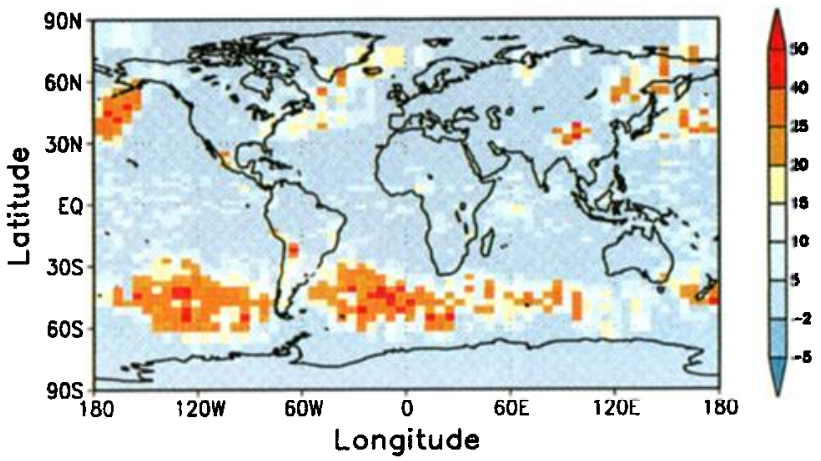

(b) Model - SNR-limited lidar

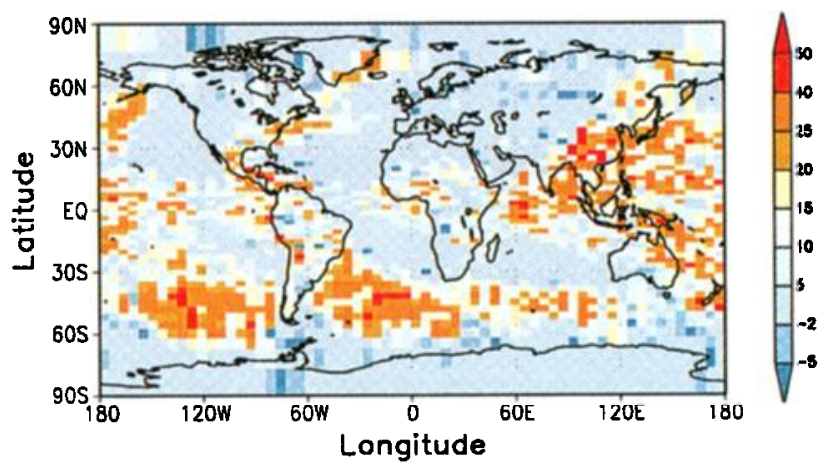

(d) Model - Radiometry, IR + VIS channel scheme

Plate 3. (a) Model cloud fraction at level 9 (about 13,200 m) and difference between this cloud fraction and the retrieved cloud fraction at the same level by (b) an SNR-limited lidar, (c) radiometry, IR channel only scheme, and (d) radiometry, IR+VIS channel scheme. Cloud fraction is retrieved by using the random cloud overlap assumption.

duced swath. Systematic errors due to the cloud diurnal cycle were not assessed in the present study.

Cirrus are poorly detected by radiometry (less than $60 \%$ ) because (1) they are spread over the two or three highest levels and (2) thin cloud top altitude is not accurately determined in multilayered cloud systems. Furthermore, when a procedure using IR channel only is considered, a significant reduction in the detection of cirrus is observed, whereas the detection of midlevel clouds is enhanced. With respect to this problem, a spaceborne lidar should lead to significant improvements in the cloud climatology of upper level clouds. About $70-80 \%$ of cirrus clouds are detected with an SNR-limited lidar, assuming the same distribution of cloudiness. The differences in upper cloud detection between an ideal and an SNR-limited lidar are due (1) to the presence at high latitudes of optically thin cirrus that would require a larger SNR (a higher number of shots and/or backscattered power would be required) and (2) to the presence of dense high level clouds at lower latitudes, which increases the transmission losses.

In our study, radiometers report more midlevel clouds than do lidars. However, some of these midlevel clouds are artificially retrieved by the radiometers in the presence of simultaneous high and low clouds. The ideal lidar and the SNR-limited lidar report a small but not insignificant fraction of the low level clouds.

While spaceborne lidar measurements are suited for vertical cloud structure retrieval, VIS and IR imagers offer the required performance for spatial cloud analysis. Therefore the combined use of these instruments should bring large improvements in the present cloud climatology.

Acknowledgments. This research has been supported by the European Space Agency (ESA) through contract number 1-2668/92/NL/CN and by the Centre National de la Recherche Scientifique (CNRS). The authors also thank two anonymous reviewers for their helpful comments.

\section{References}

Barkstrom, B. R., Clouds and the Earth's radiant energy system science team meeting, Earth Obs., 9, 12-16, 1997. 
Baum, B. A., R. F. Arduini, B. A. Wielicki, P. Minnis, and S.-C. Tsay, Multilevel cloud retrieval using multispectral HIRS and AVHRR data: Nighttime oceanic analysis, $J$. Geophys. Res., 99, 5499-5514, 1994.

Baum, B. A., T. Uttal, M. Poellot, T. P. Ackerman, J. M. Alvarez, J. Intrieri, D. O'C. Starr, J. Titlow, V. Tovinkere, and E. Clothiaux, Satellite remote sensing of multiple cloud layers, J. Atmos. Sci., 52, 4210-4230, 1995.

Bony, S., H. Le Treut, J.-P. Duvel, and R. Kandel, Satellite validation of GCM-simulated annual cycle of the Earth radiation budget and cloud forcing, J. Geophys. Res., 97, 18,061-18,081, 1992.

Brown, S. C., A. J. Illingworth, A. J. Heymsfield, C. M. McFarquhar, K. A. Browning, and M. Gosset, The role of spaceborne millimeter-wave radar in global monitoring of ice cloud, J. Appl. Meteorol., 34, 2346-2366, 1995.

Cairns, B., Diurnal variations of cloud from ISCCP data, Atmos. Res., 37, 133-146, 1995.

Charlock, T. P., F. Rose, T. Alberta, G. L. Smith, D. Rutan, N. Manalo-Smith, P. Minnis, and B. Wielicki, Cloud profiling radar requirements: Perspective from retrievals of the surface and atmospheric radiation budget and studies of atmospheric energetics, in Utility and Feasibility of a Cloud Profiling Radar: Report of GEWEX Topical Workshop (Pasadena, California, USA 29 June-1 July 1993), Tech. Doc. WMO/TD 593, pp. B10-B21, 1994.

Chou, M. D., Surface radiation in the tropical Pacific, $J$. Clim. Appl. Meteorol., 24, 83-92, 1985.

Del Genio, A. D., Accuracy requirements, in Long-Term Monitoring of Global Climate Forcings and Feedbacks, edited by J. Hansen, W. Rossow, and I. Fung, pp. 1319, NASA GISS, 1992.

Del Genio, A. D., and M.-S. Yao, Properties of deep convective clouds in the ISCCP pilot data set, paper presented at 17th Conference on Hurricanes and Tropical Meteorology, Am. Meteorol. Soc., 1987.

Doutriaux, M., H. Le Treut, and G. Sèze, Une étude de la couverture nuageuse : Comparaison des climatologies issues de l'observation satellitaire, des observations au sol, et de modèles de circulation générale du LMD, LMD Tech. Rep. 196, [Available from Lab. de Météorol. Dyn., Ecole Normale Supérieure, Paris, France], 1995a.

Doutriaux, M., H. Le Treut, and G. Sèze, Different versions of the LMD GCM and their validation through ISCCP analysis, in Proceedings of the First International AMIP Scientific Conference, edited by W. L. Gates, Tech. Doc. WMO/TD 732, pp. 343-352, World Meteorol. Org., Geneva, Switzerland, 1995b.

Flesia, C., A. V. Starkov, and Y. Emery, Lidar signal retrieval under low signal to noise ratio conditions, Eur. Space Agency/Eur. Space Res. and Tech. Cent., 197 pp., 1996.

Fouquart, Y., and B. Bonnel, Computations of solar heating of the Earth's atmosphere: A new parameterization, Beitr. Phys. Atmos., 53, 35-62, 1980.

Han, Q., W. B. Rossow, and A. A. Lacis, Near-global survey of droplet radii in liquid water cloud using ISCCP data, J. Clim., 7, 465-497, 1994.

Hansen, J., Climsat rationale, in Long-Term Monitoring of Global Climate Forcings and Feedbacks, edited by J. Hansen, W. Rossow, and I. Fung, pp. 26-35, NASA GISS, 1992.

Le Treut, H., and Z. X. Li, Sensitivity of an atmospheric general circulation model to prescribed SST changes: Feedback effects associated with the simulation of cloud optical properties, Clim. Dyn., 5, 175-187, 1991.

Liao, X., W. B. Rossow, and D. Rind, Comparison between SAGE II and ISCCP high-level clouds, 1, Global and zonal mean cloud amounts, J. Geophys. Res., 100, 1121-1135, 1995a.
Liao, X., W. B. Rossow, and D. Rind, Comparison between SAGE II and ISCCP high-level clouds, 2, Locating cloud tops, J. Geophys. Res., 100, 1137-1147, 1995b.

Liou, K., Influence of cirrus clouds on weather and climate processes: A global perspective, Mon. Weather Rev., 114 1167-1197, 1986.

McCormick, M. P., et al., Scientific investigations planned for the Lidar in-Space Technology Experiment (LITE), Bull. Am. Meteorol. Soc., 74, 205-214, 1993.

Mokhov, I. I., O. P. Skrotskaya, and I. G. Ostapenko, On the overlapping of clouds of different layers in general circulation models, Izr. Russ. Acad. Sci., Atmos. Oceanic Phy., Engl. Transl., 30, 527-532, 1995.

Morcrette, J.-J., Radiation and cloud radiative properties in the European Centre for Medium Range Weather Forecasts forecasting system, J. Geophys. Res., 96, 9121-9132, 1991.

Pelon, J., et al., A study of the potential contribution of a backscatter lidar to climatological studies: Assessment of the contribution of a spaceborne backscatter lidar to WRCP, Eur. Space Agency, 137 pp., 1996.

Platt, C. M. R., Lidar and radiometric observations of cirrus clouds, J. Atmos. Sci., 30, 1191-1203, 1973.

Platt, C. M. R., Remote sounding of high clouds, 1, Calculation of visible and infrared optical properties from lidar and radiometer measurements, J. Appl. Meteorol., 18 1130-1143, 1979.

Platt, C. M. R., and G. L. Stephens, The interpretation of the remotely sensed high cloud emittances, J. Atmos. Sci., 37, 2314-2322, 1980.

Randall, D. A., Harshvardhan, D. A. Dazlich, and T. G. Corsetti, Interactions among radiation, convection, and large-scale dynamics in a general circulation model, $J$. Atmos. Sci., 46, 1943-1970, 1989.

Rossow, W. B., Effects of sampling on satellite measurements of clouds, in Utility and Feasibility of a Cloud Profiling Radar: Report of GEWEX Topical Workshop (Pasadena, California, USA 29 June-1 July 1993), Tech. Doc. WMO/TD 593, pp. B58-B64, 1994.

Rossow, W. B., and A. A. Lacis, Global, seasonal cloud variations from satellite radiance measurements, 2 , Cloud properties and radiative effects, J. Clim., 3, 1204-1253, 1990.

Rossow, W. B., and R. A. Schiffer, ISCCP cloud data products, Bull. Am. Meteorol. Soc., 72, 2-20, 1991.

Rossow, W. B., L. C. Garder, and A. A. Lacis, Global, seasonal cloud variations from satellite radiance measurements, 1, Sensitivity of analysis, J. Clim., 2, 419-458, 1989.

Rossow, W. B., L. C. Garder, P. J. Lu, and A. W. Walker, International Satellite Cloud Climatology Project (ISCCP): Documentation of cloud data, Tech. Doc. WMO/TD 266, 76 pp. +2 append., World Meteorol. Org., Geneva, Switzerland, 1991.

Rossow, W. B., A. W. Walker, and L. C. Garder, Comparison of ISCCP and other cloud amounts, J. Clim., 6, 2394-2418, 1993.

Sadourny, R., and K. Laval, January and July performance of the LMD general circulation model, in New Perspectives in Climate Modelling, edited by A. Berger and C. Nicolis, pp. 173-198, Elsevier, New York, 1984.

Schiffer, R. A., and W. B. Rossow, The International Satellite Cloud Climatology Project (ISCCP): The first project of the World Climate Research Program, Bull. Am. Meteorol. Soc., 64, 779-784, 1983.

Sheu, R.-S., J. Curry, and G. S. Liu, Vertical stratification of tropical cloud properties as determined from satellite, J. Geophys. Res., 102, 4231-4245, 1997.

Slingo, A., and J. M. Slingo, The response of a general circulation model to cloud longwave forcing, I, Introduc- 
tion and initial experiment, Q. J. R. Meteorol. Soc., 114 1027-1062, 1988.

Spinhirne, J. D., and W. D. Hart, Cirrus structure and radiative parameters from airborne lidar and spectral radiometer observations: The 28 October 1986 FIRE study, Mon. Weather Rev., 118, 2329-2343, 1990.

Spinhirne, J. D., M. Z. Hansen, and L. O. Caudill, Cloud top remote sensing by airborne lidar, Appl. Opt., 22, $1564-1571,1982$.

Stubenrauch, C. J., W. B. Rossow, F. Cheruy, N. A. Scott, and A. Chedin, Combining 3I cloud parameters and ISCCP for better understanding of cloud radiative effects, paper presented at International Radiation Symposium, Fairbanks, Alaska, 1996.

Tian, L., and J. A. Curry, Cloud overlap statistics, $J$. Geophys. Res., 94, 9925-9935, 1989.

Wang, P.-H., P. Minnis, M. P. McCormick, G. S. Kent, and K. M. Skeens, A 6-year climatology of cloud occurrence frequency from Stratospheric Aerosol and Gas Experiment II observations (1985-1990), J. Geophys. Res., 101, 29,407-29,429, 1996.

Warren, S. G., C. J. Hahn, and J. London, Simultaneous occurrence of different cloud types, J. Clim. Appl. Meteorol., 24, 658-667, 1985.

Warren, S. G., C. J. Hahn, J. London, R. M. Chervin, and R. L. Jenne, Global distribution of total cloud cover and cloud type amounts over land, NCAR Tech. Note TN$273+S T R, 29$ pp. +200 maps, Natl. Cent. for Atmos. Res., Boulder, Colo., Oct. 1986.

Warren, S. G., C. J. Hahn, J. London, R. M. Chervin, and R. L. Jenne, Global distribution of total cloud cover and cloud type amounts over the ocean, NCAR Tech. Note
$T N-317+S T R, 42$ pp. +170 maps, Natl. Cent. for Atmos. Res., Boulder, Colo., Dec. 1988.

Winker, D. M., R. H. Couch, and M. P. McCormick, An overview of LITE: NASA's Lidar In-space Technology Experiment, Proc. IEEE, 84, 164-180, 1996.

Yu, W., M. Doutriaux, G. Sèze, H. Le Treut, and M. Desbois, A methodology study of the validation of clouds in GCMs using ISCCP satellite observations, Clim. Dyn., 12, 389-401, 1996.

Zhang, Y. C., W. B. Rossow, and A. A. Lacis, Calculation of surface and top of the atmosphere radiative fluxes from physical quantities based on ISCCP data sets, 1, Method and sensitivity to input data uncertainties, J. Geophys. Res., 100, 1149-1165, 1995.

M. Desbois and P. Flamant, Laboratoire de Météorologie Dynamique, Ecole Polytechnique, 91128 Palaiseau Cedex, France.

M. Doutriaux-Boucher, Laboratoire d'Optique Atmosphérique, Université de Lille-l, 59655 Villeneuve d'Ascq Cedex, France. (e-mail: mdoutri@loa.univ-lille1.fr)

H. Le Treut and G. Sèze, Laboratoire de Météorologie Dynamique, Tour 15-25, Boîte 99, CNRS/UPMC, 4 place Jussieu, 75252 Paris Cedex 05, France. (e-mail: letreut@lmd.jussieu.fr; seze@lmd.jussieu.fr)

J. Pelon and V. Trouillet, Service d'Aéronomie, Tour 1525, Boîte 102, CNRS/UPMC, 4 place Jussieu, 75252 Paris Cedex 05, France. (e-mail: jacques.pelon@aero.jussieu.fr)

(Received January 28, 1998; revised June 29, 1998; accepted July 7,1998 .) 